\title{
e-interview
}

columns

\section{Vikram Patel}

Vikram Patel is a Senior Lecturer at the London School of Hygiene \& Tropical Medicine (www.lshtm.ac.uk) and Chairperson, The Sangath Society, Goa, India (www.sangath.com). He trained in KEM Hospital (Mumbai) and the Maudsley Hospital/Institute of Psychiatry, London. e-mail: vikpat@goatelecom.com

If you were not a psychiatrist, what would you do?

I would have liked to be a chef, an actor, a travel writer or a photographer (in that order!)

What has been the greatest impact of your profession on you personally? The feeling of tremendous reward when someone with a mental illness recovers.

Do you feel stigmatised by your profession?

I did when I first chose psychiatry as a fresh medical graduate. However, now, perhaps because psychiatry is gaining much greater public acceptance, I feel no stigma at all.

\section{What are your interests outside of} work?

Eating, anything to do with a warm ocean and travelling.

\section{What job gave you the most useful} training experience?

My two years in the University of Zimbabwe Medical School exposed me to my first real dose of psychiatry in an under-resourced setting. The experiences there were the most formative ones in guiding my work today. In the past few years, I have worked with a number of non-governmental organisations in India, and this experience has exposed me to the role of this unique sector in innovating new services and implementing relevant research programs.

Which book/text has influenced you most?

David Werner's WhereThere Is No Doctor for its style and goal to make health care accessible to all, and Arthur Kleinman's Patients and Healers in the Context of Culture for its seminal contribution to our

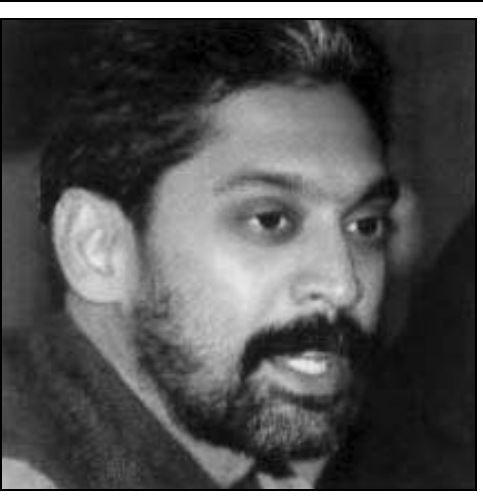

Greater external monitoring and regulation by a multi-disciplinary body.

What conflict of interest do you encounter most often?

Whether or not to accept apparently innocuous drug company generosity, such as dinners and pens.

Do you think psychiatry is brainless or mindless?

Not at all! I find it one of the most intellectually stimulating of all the medical sciences. But perhaps less stimulating than cooking and travelling!

How would you entice more medical students into the profession?

understanding of cultural influences on mental disorders.

What part of your work gives you the most satisfaction?

Building capacity, particularly creating a public health perspective among mental health professionals, and creating a mental health perspective among public health professionals. I particularly enjoy working in settings where mental health specialists are few and far between because I find that the challenges of meeting mental health needs in these settings provides a fertile ground for spawning exciting new innovations.

What is the most promising opportunity facing the profession?

The greater global awareness and acknowledgement that mental illnesses are real causes of suffering everywhere, and that much can be done to help people with mental illnesses. Of course, there is still a long way to go as far as awareness is concerned, but tremendous progress has been made in the past decade.

What is the greatest threat?

Psychiatry itself! It has become overcomplicated, too far removed from one of its key goals of improving mental health in the community, and in many countries, hijacked by pharmacologically driven professional societies.

What single change would substantially improve quality of care?
Reduce the jargon, make it more relevant to general practice, and integrate most of psychiatric training into general medicine.

What are the main ethical problems that psychiatrists will face in the future?

The issue of drug costs as many developing countries are being coerced to signTRIPS (trade related aspects of intellectual property rights), and psychiatric disorders are not considered excuses for purchasing low cost drugs.

How would you improve clinical psychiatric training?

Include an overseas training period; what you can learn by spending one year in an under-resourced setting is worth several years in the NHS. Besides, it can help change your perspective about being understaffed on your NHS job when you work in a place where there are no psychiatrists at all.

What single area of psychiatric research should be given priority? I am biased here, of course, but psychiatric research in low and middle income countries is the key to enabling a truly international psychiatry. Not only would this serve psychiatry as a global medical discipline, but also the needs of the vast majority of the global population of those with mental disorders who live in low and middle income countries. 